Reversible hydration of $\alpha$-dicarbonyl compounds

from ab initio metadynamics simulations:

comparison between pyruvic and glyoxylic acids

\title{
in aqueous solution
}

\author{
Rodolphe Pollet* ${ }^{*}$ and Wutharath Chin ${ }^{\ddagger}$ \\ †Université Paris-Saclay, CEA, CNRS, NIMBE, 91191, Gif-sur-Yvette, France. \\ ‡Université Paris-Saclay, CNRS, Institut des Sciences Moléculaires d'Orsay, 91405, Orsay, \\ France
}

E-mail: rodolphe.pollet@cea.fr

Phone: +33 (1)690 83713 


\begin{abstract}
Glyoxylic and pyruvic oxoacids are widely available in the atmosphere as gas phase clusters, particles or in wet aerosols. In aqueous conditions, they undergo interconversion between the unhydrated oxo and the gem-diol forms, where two hydroxyl groups replace the carbonyl group. We here examine the hydration equilibrium of glyoxylic and pyruvic acids with first-principle simulations in water at ambient conditions, using ab initio metadynamics to reconstruct the corresponding free-energy landscapes. The main results are as follows: (i) our simulations reveal the high conformational diversity of these species in aqueous solution. (ii) We show that the gem-diol is strongly favored in water compared to its oxo counterpart by $29 \mathrm{~kJ} / \mathrm{mol}$ and $16 \mathrm{~kJ} / \mathrm{mol}$ for glyoxylic and pyruvic acids, respectively. (iii) From our atomic scale simulations, we present new insights for the reaction mechanisms with a special focus on hydrogen-bonds arrangements and the electronic structure of the transition state.
\end{abstract}

\title{
Introduction
}

Water-soluble organic acids are highly abundant in atmospheric aerosols and among these, carboxylic acids are prevalent. These volatile organic compounds contribute to the formation of secondary organic aerosols ${ }^{1,2}$ and thus have profound effects on the climate by absorbing or reflecting solar radiation, ${ }^{3}$ and modifying cloud formation. ${ }^{4}$ They also impact human health since they form particulate matter present in urban pollution. ${ }^{5}$

Among these organic acids, $\alpha$-dicarbonyls are widely found in atmospheric aerosols, marine aerosols, cloud and surface water. ${ }^{6-9}$ They are common products of the oxidation of biogenic emission such as isoprene. ${ }^{10,11}$ Being intermediate products they have attracted considerable interest in photochemistry and oxidation studies. $\alpha$-dicarbonyls encounter a very complex chemistry that is multiphase, e.g., occurring in aqueous, gas and particle phases. ${ }^{12-15}$ Understanding their chemical reactivity would thus be valuable to environmental chemistry. 
One important and well-known class of aqueous-phase reactions is the hydration-dehydration equilibrium of $\alpha$-ketoacids (also referred to as $\alpha$-oxoacids or 2 -oxocarboxylic acids). The interconversion of keto to gem-diol forms in water strongly impacts the role of organic acids and consequently of secondary organic aerosols. ${ }^{1}$ It is thus of crucial importance to consider the relevant chemical structure because it will dictate the physical chemistry and reactivity of the compound, e.g., the proneness to form oligomers, ${ }^{7,16}$ the role in oxidation and in photochemical processes. ${ }^{12,17-19}$

Glyoxylic acid and pyruvic acid are the most abundant 2-oxocarboxylic acids in the atmosphere ${ }^{20,21}$ and are widely present in aqueous secondary organic aerosols. ${ }^{10}$ In the gas phase they both have four stable rotamers that differ by the trans/cis configurations, with the uppercase letter $(T, C)$ referring to OCCO dihedral angle and the lowercase letter $(t, c)$ to $\mathrm{CCOH}$. The two most stable conformers of glyoxylic and pyruvic acids are named $T c$ and $T t$, the others being higher in energy. ${ }^{22-27}$ Aqueous-phase $\alpha$-oxoacids can exist in the keto form, i.e., dehydrated, or in the hydrated gem-diol form. According to their acid-base equilibrium the aldehyde and gem-diol forms of glyoxylic acid $\left(\mathrm{p} K_{a}=3.2-3.3\right),{ }^{28,29}$ and the keto and diol forms of pyruvic acid $\left(\mathrm{p} K_{a}=1.7-2.2\right),{ }^{26,30,31}$ can be protonated or deprotonated in solution (i.e. neutral or anionic form) (Scheme 1). So in total four speciated compounds may co-exist in the aqueous phase for each $\alpha$-oxoacid. The abundance of the aldehyde or keto and diol forms is dependent on the $\mathrm{pH}:{ }^{27,32}$ Aldehydic and keto forms are favoured at higher $\mathrm{pH}$, so are anions, and diol forms as well as protonated forms are predominant at lower pH. $\alpha$ dicarbonyls may also be present as enolic forms but they are minor in solution or calculated to be less stable. ${ }^{31,33}$ The equilibrium reactions in the aqueous phase between glyoxylic and its gem-diol, 2,2-dihydroxyacetic acid, and between pyruvic and 2,2-dihydroxypropionic acid have been widely investigated experimentally using UV spectrophotometry, ${ }^{27,30,31,34}$ relaxation methods, ${ }^{35}$ chemical reactions, ${ }^{28}$ and NMR techniques. ${ }^{26,28,31,36-39}$

In order to access the atomic scale details to describe the hydration and dehydration reactions, theoretical calculations can be used to complement experiments. The simplest 
<smiles>[R]C(=O)C(=O)O</smiles><smiles>C=[Tl]</smiles><smiles>[R]C(=O)C(=O)Cl</smiles>
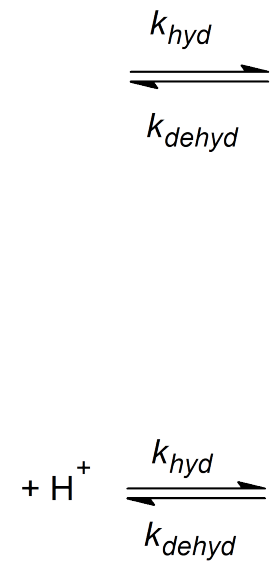<smiles>[R]C(O)(O)C(=O)O</smiles>
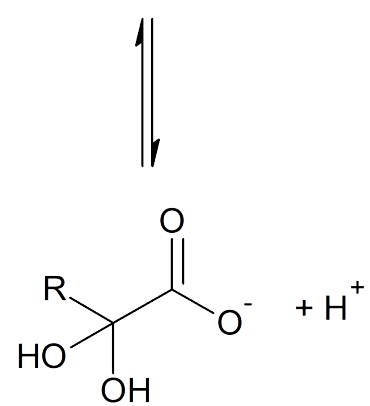

Scheme 1: The aldehyde/keto and gem-diol forms of glyoxylic $(\mathrm{R}=\mathrm{H})$ and pyruvic $\left(\mathrm{R}=\mathrm{CH}_{3}\right)$ acids. The vertical reaction depicts the acid-base equilibrium between the protonated and deprotonated forms of the acids and are characterized by their pKa values.

approach to model the addition of water on glyoxylic and pyruvic acids relies on ab initio calculations including only a few explicit water molecules to obtain their microsolvation structures. While these studies reveal which conformers are the most stable and provide useful information on the microscopic arrangement of hydrogen bonds, ${ }^{23,24,27,40-43}$ they lack entropic effects at finite temperature. A further improvement consists of calculations using an approximate and mixed treatment of these bulk solvent effects with a dielectric continuum surrounding a first shell of explicit water molecules. ${ }^{33}$ However the most realistic method is to perform ab initio molecular dynamics (AIMD) simulations at finite temperature of a periodic cell filled with the solute and a large number of explicit solvent molecules. ${ }^{44}$ An AIMD study of formaldehyde and methanediol in aqueous solution showed that the entropic contribution can be very important. ${ }^{45}$ In the present work, we use a similar approach, relying on biased AIMD simulations to accelerate the observation of rare events (see also a pioneering work on the acid-catalyzed addition of water on formaldehyde ${ }^{46}$ ) in water at $300 \mathrm{~K}$. While Ref. 45 reported the results of the Blue Moon method with only one constraint applied to dehydration, our approach relies on the metadynamics method with two collective variables (see below) applied to the reversible reaction, giving a more complete view of the free- 
energy landscape, hence the reaction paths. In this article we first provide thermodynamical and structural information for glyoxylic and pyruvic acids, separately, from the free-energy landscapes that describe the interconversion of the dehydrated and hydrated species. We then explore the reaction mechanisms thanks to a fruitful comparison between both acids. Finally, we comment on the implications of our simulations on atmospheric chemistry.

\section{Computational details}

The two Car-Parrinello (CP) simulations ${ }^{44,47}$ reported in this paper were based on planewave DFT calculations using the PBE exchange-correlation approximation. ${ }^{48}$ In order to reduce the computational cost, they relied on large core ultrasoft pseudopotentials ${ }^{49}$ including scalar relativistic effects and allowing a cutoff energy of only 30 Ry. A geometry optimization in aqueous solution was first performed to prepare the system modeled by a $12.42 \AA$ cubic box including 62 water molecules that surround the $\alpha$-dicarbonyl compound (with $T c$ as the initial conformation). It was followed by an unbiased CP simulation with a fictitious electron mass of 700 a.u., a time step of 6 a.u., and Nosé-Hoover chain thermostats (with a chain length of three) both for ions (with the target temperature set to $300 \mathrm{~K}$ ) and electrons in order to prevent heat transfer between both subsystems. Note that hydrogen atoms were also replaced by deuterium atoms to maintain adiabaticity. For glyoxylic and pyruvic acids in aqueous solution, the time lengths of these CP trajectories were 65 and 34 ps, respectively. During these two preliminary simulations, deprotonation of the carboxyl group (i.e., jump of the carboxylic proton onto a H-bond acceptor water molecule) occurred spontaneously after approximately 2 and 5 ps for glyoxylic and pyruvic acids, respectively. Note that such time-dependent changes can only be analyzed during non-biased simulations whereas only thermodynamical data can be extracted from metadynamics simulations. Therefore we use the Eyring equation in the Results and Discussion section to convert experimental reaction rates to free-energy barriers. Other theoretical approaches can provide 
reaction rates but require statistics currently out of reach of ab initio simulations. ${ }^{50}$ Because of these two events, the next metadynamics simulations started with solvated glyoxylate and pyruvate anions (or at least pyruvic acid sharing its proton with a neighbouring water molecule), respectively, in acidic solution (due to the presence of $\mathrm{H}_{3} \mathrm{O}^{+}$in the simulation box). Therefore, intramolecular hydrogen bonds as observed in the initial Tc conformer can not occur anymore. Interestingly, as the proton of the carboxylic group detaches, the pyruvate becomes significantly twisted, with an average $\mathrm{OCCO}(\mathrm{H})$ dihedral angle changing from -19 to -100 degrees (Figure 1). This is obviously caused by the repulsion between the carboxylate and carbonyl groups.

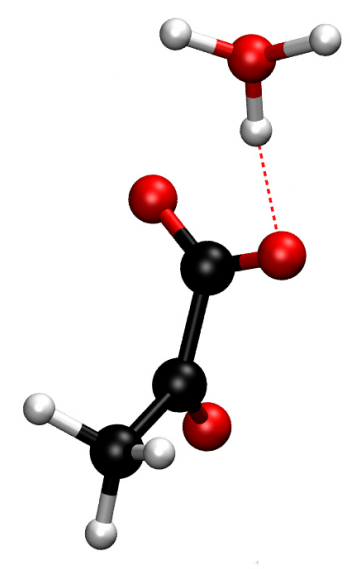

Figure 1: Configuration (other solvent molecules not shown) from the preliminary CP trajectory with both (twisted) pyruvate and H-bonded water molecule sharing the carboxyl hydrogen.

In order to describe the reversible hydration of glyoxylic and pyruvic acids, ab initio metadynamics ${ }^{51-56}$ simulations were then performed by selecting two collective variables (CVs), that enable the two-dimensional free-energy landscape to be explored. CV1 was taken as the coordination numbers of $O_{\text {carbonyl }}$ with respect to all water protons and CV2 as those of $C_{\text {carbonyl }}$ with respect to all water oxygens. Both collective variables were chosen to accelerate the formation of the geminal hydroxyl groups of the diol. For both coordination 
numbers, the following expression was used: ${ }^{57,58}$

$$
C N=\sum_{i} \frac{1-\left(d_{i} / d_{0}\right)^{p}}{1-\left(d_{i} / d_{0}\right)^{p+q}}
$$

where $d_{i}$ is the distance between the $O_{\text {carbonyl }}$ and the $i^{\text {th }}$ hydrogen $\left(d_{0}=1.4 \AA\right)$ or between $C_{\text {carbonyl }}$ and the $i^{\text {th }}$ oxygen $\left(d_{0}=1.8 \AA\right), p=6$ and $q=6$. This set of parameters was chosen to discriminate between reactants and products and was already used in a similar study on chemical reactions of small organic molecules. ${ }^{59}$ In addition, an extended Lagrangian method was used to slowly propagate auxiliary variables dragging these two CVs away thanks to a harmonic coupling, ${ }^{58,60}$ with coupling constants $k$ set to 2.0 and fictitious masses to 50 a.u. The width and height of the Gaussian functions (or hills) accumulated to reconstruct the free-energy hypersurface were set to 0.08025 and 0.001 a.u. respectively (i.e., approximately $\left.1 k_{B} T\right)$ up to a point where the $\mathrm{O}$ atom of water could attach onto the $C_{\text {carbonyl }}$ atom of the acid, where the hill height had changed to $1 / 4 k_{B} T$. The metadynamics time step was actually not fixed. Instead, it was determined on-the-fly to avoid "hill surfing". ${ }^{55}$ Moreover the temperature of the auxiliary variables was bound to fluctuate in the $300 \pm 200 \mathrm{~K}$ range thanks to a simple scaling of the velocities. Note that a third CV to describe the associated acid-base reactions was not included to reduce the computational time. As a consequence, the neutral and anionic forms share the same free-energy basin. A structural analysis within each region should reveal if one form actually dominates or if a mixture exists, as detailed later.

For glyoxylic acid, the metadynamics simulation was stopped when the hydroxyl group of the gem-diol originating from $O_{\text {aldehyde }}$ suddenly detached (making both CVs not relevant anymore), namely after 3050 hills. Along this trajectory, the formation of the diol was sampled twice. The final breaking of the $\mathrm{C}-\mathrm{O}$ bond seems to be caused by the attack of two H-bond donor water molecules and one H-bond acceptor water molecule with respect to this hydroxyl group of the gem-diol. 
In the case of pyruvic acid, 2661 hills were accumulated during the metadynamics simulation. Sampling of the free-energy landscape included several formation events of the gem-diol and dehydration processes before reaching a barrierless regime. It is also worth mentioning that the enolization of the keto form was not observed during the simulation.

\section{Results and discussion}

\section{Glyoxylic acid: thermodynamics and structure}

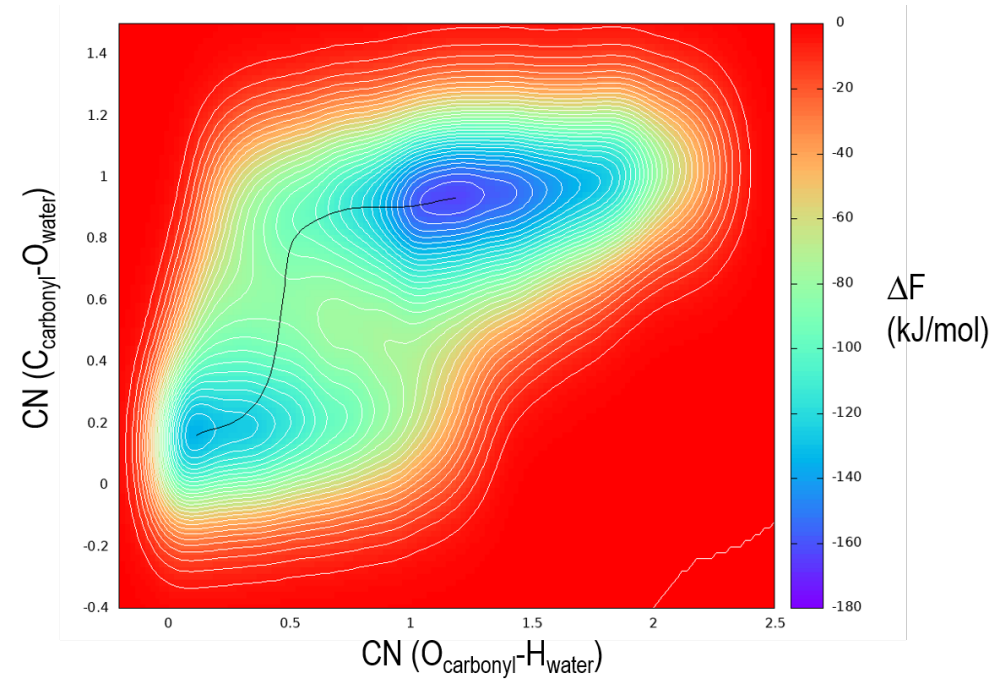

Figure 2: Free energy hypersurface (in $\mathrm{kJ} / \mathrm{mol}$ ) obtained for glyoxylic acid. CV1 (coordination number of $\mathrm{O}_{\text {carbonyl }}$ with respect to water hydrogens) as x-axis and CV2 (coordination number of $\mathrm{C}_{\text {carbonyl }}$ with respect to water oxygens) as y-axis. The lowest free-energy pathway is shown.

The two-dimensional (CV1 and CV2) free-energy landscape was reconstructed by the metadynamics algorithm (Figure 2). It exhibits two basins centered around the $(0.11,0.16)$ and $(1.18,0.94)$ collective coordinates, as expected since a coordination number equal to zero corresponds to an isolated atom whereas a value close to one is obtained when part of a water molecule attaches. The $(0.11,0.16)$ coordinates indicate that there is no close interaction between the carbonyl group and water molecules in the first basin. In contrast both the oxygen and carbon of the carbonyl are being attacked by water in the second basin. 
These two minima mainly correspond to the glyoxylate anion and the hydrated gem-diol form of glyoxylic acid (see below).

Table 1: Summary of the calculated free-energy differences (in $\mathrm{kJ} / \mathrm{mol}$ ) from metadynamics simulations for glyoxylic and pyruvic acids in water at $300 \mathrm{~K}$

\begin{tabular}{lcc}
\hline Free-energy & Glyoxylic acid & Pyruvic acid \\
\hline$\Delta F_{1 \rightarrow 2}^{\ddagger}{ }^{a}$ & 52 & 68 \\
$\Delta F_{2 \rightarrow 1}^{\ddagger}{ }^{b}$ & 81 & 84 \\
$\Delta F_{12}{ }^{c}$ & 29 & 16 \\
\hline
\end{tabular}

${ }^{a}$ Free-energy barrier from first (unhydrated form) to second basin (gem-diol); ${ }^{b}$ Free-energy barrier from second (gem-diol) to first (unhydrated form) basin; ${ }^{c}$ Free-energy difference between minima of first (unhydrated form) and second basin (gem-diol).

According to this simulation, the diol is $29 \mathrm{~kJ} / \mathrm{mol}$ more stable than the unhydrated aldehyde form (see Table 1), which is in agreement with the high value of the equilibrium constant of hydration ( $K_{H}$ of 1100 in Ref. 19). After reconstructing the lowest free-energy pathway using the recipe of Ensing et al., ${ }^{55}$ the transition state is found at $(0.40,0.60)$. The barrier from the first basin to the transition state is estimated to $52 \mathrm{~kJ} / \mathrm{mol}$, while it requires $81 \mathrm{~kJ} / \mathrm{mol}$ to escape from the second basin.

In order to confirm which species were present in the first and second free-energy basins, two structural analyses were carried out by selecting configurations for which the collective variables fall within bounded intervals. Respectively 11089 and 40217 configurations were taken into account. From the small peak around $1 \AA$ of the (carboxyl)oxygen-(water)hydrogen radial distribution function (RDF) (Figure S1) as well as the structures observed from the trajectory, we can conclude that the first free-energy basin corresponds to the glyoxylate anion, which means that the $\mathrm{pH}$ in our simulation is higher than the pKa.

For the second basin, a mixture of deprotonated and protonated gem-diol is found as displayed by the peak at around $1 \AA$ in the RDF of Figure S1. As stated above, this degeneracy stems from the lack of a third collective variable to describe the acid-base reaction. The fact that a pure anion is observed in the aldehyde form whereas a mixture of anionic and neutral form is found for the diol can be explained by the stronger acidity of the oxo species. 
Comparing our calculated free-energy barriers with experiment is not straightforward since our second basin contains a mixture of anionic and neutral forms. However, we can tentatively compare our barrier of hydration $(52 \mathrm{~kJ} / \mathrm{mol})$ with the experimental value deduced through the Eyring equation from Sørensen et al. for glyoxylate, namely $79 \mathrm{~kJ} / \mathrm{mol}$ (from a hydration rate constant $k_{h y d}$ of $0.083 \mathrm{~s}^{-1}$ at $298 \mathrm{~K}$ and $\mathrm{pH}=7$ ) ${ }^{28}$ By following Doussin and Monod and using an equilibrium constant $K_{H}$ of 67 instead of 15, it decreases slightly to $75 \mathrm{~kJ} / \mathrm{mol}$ (from $k_{h y d}$ of $0.42 \mathrm{~s}^{-1}$ at $298 \mathrm{~K}$ and $\mathrm{pH}=7$ ). ${ }^{19}$ Note that for the neutral form, the reported value is even lower at $65 \mathrm{~kJ} / \mathrm{mol}$ (from $k_{\text {hyd }}$ of $27.5 \mathrm{~s}^{-1}$ at $298 \mathrm{~K}$ and $\mathrm{pH}=7) \cdot{ }^{19}$ As for the dehydration, the agreement with the experimental data is better. Our estimated free-energy barrier of dehydration of $81 \mathrm{~kJ} / \mathrm{mol}$ is very close to the experimental barriers found for the neutral $\left(82 \mathrm{~kJ} / \mathrm{mol} \text { from } k_{\text {dehyd }} \text { of } 0.0250 \mathrm{~s}^{-1} \text { at } 298 \mathrm{~K} \text { and } \mathrm{pH}=7\right)^{19,28}$ and for the anion $\left(85 \mathrm{~kJ} / \mathrm{mol}\right.$ from $k_{\text {dehyd }}$ of $0.0064 \mathrm{~s}^{-1}$ at $298 \mathrm{~K}$ and $\left.\mathrm{pH}=7\right) .{ }^{19}$ The rate constants are summarized in Table S1. Our simulation therefore confirms that hydration is faster than dehydration ${ }^{28}$ and that the hydrated form is more stable. For a quantitative improvement, our theoretical study would require a more accurate exchange-correlation density functional approximation, ${ }^{61}$ a larger simulation cell with more solvent molecules, more stringent metadynamics parameters, and the calculation of the transmission coefficient in the Eyring equation (to compare directly with the experimental rate constants).

We now proceed with the structural analysis of the gem-diol. As mentioned above, the metadynamics trajectory inside the second basin reveals a mixture of deprotonated and protonated forms. Moreover, in the latter case, both cis and trans conformations of the $\mathrm{CC}(\mathrm{OH})_{\text {carboxyl }}$ dihedral angle are observed (Figure 3). In all cases, the two hydroxyl groups point in different directions, one outward and up and the other outward and down, while no symmetrical configuration of the hydroxyls is found. This can be observed on the hydrogen(hydroxyl)hydrogen RDF (Figure 4), which exhibits two peaks at approximately 2.25 and $2.85 \AA$ corresponding to these two nonsymmetrical configurations. Besides, our simulation shows that the hydroxyl groups can form three hydrogen bonds with the solvent molecules 

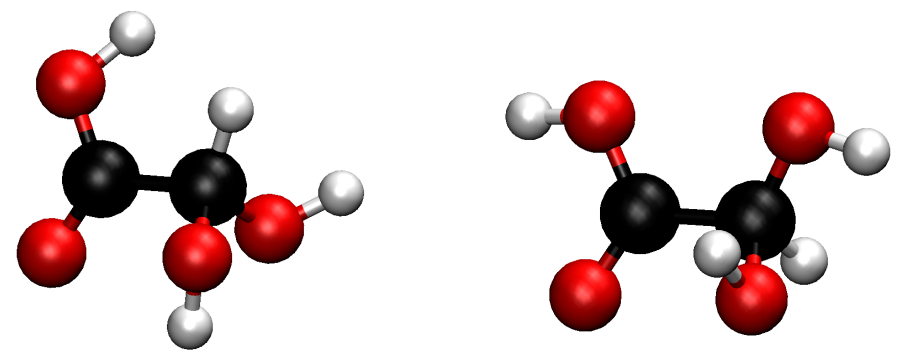

Figure 3: Configurations showing a gem-diol of glyoxylic acid in a cis (left) and trans (right) conformations with the two hydroxyl groups attached to $\mathrm{C}^{\alpha}$ atom pointing in outward up and down directions. Solvent molecules are not shown.

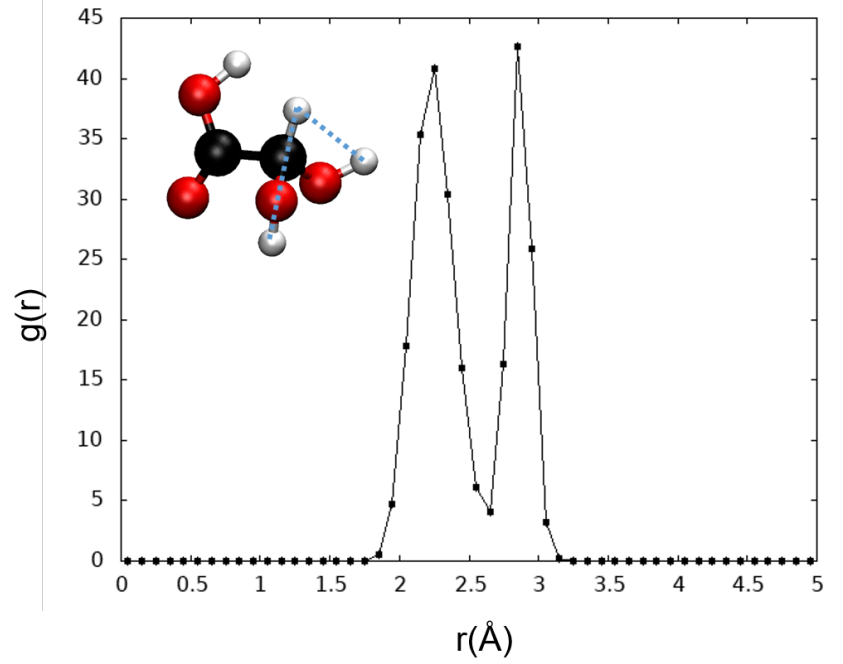

Figure 4: Hydrogen-(hydroxyl)hydrogen RDF within the second basin (gem-diol) in the case of glyoxylic acid. X-axis units in $\AA$. Example of a gem-diol structure as inset. Dashed lines indicate the distances computed for the RDF calculations. 
with an approximate (hydroxyl)oxygen-(water)oxygen distance of $2.65 \AA$ (Figure 5). More precisely, there are two donor water molecules plus one acceptor water molecule around each hydroxyl group (hence a number integral of RDF of 2.96). Consequently, the average length of the $\mathrm{OH}$ covalent bond is $1.02(0.03) \AA$. It is longer than the bond distance computed for the gas-phase diol (0.963 and $0.968 \AA) .{ }^{23}$ In addition, this description based on our fully explicit solvent model provides useful hints regarding the minimum number of water molecules to be included in theoretical studies restrained to a few explicit water molecules.

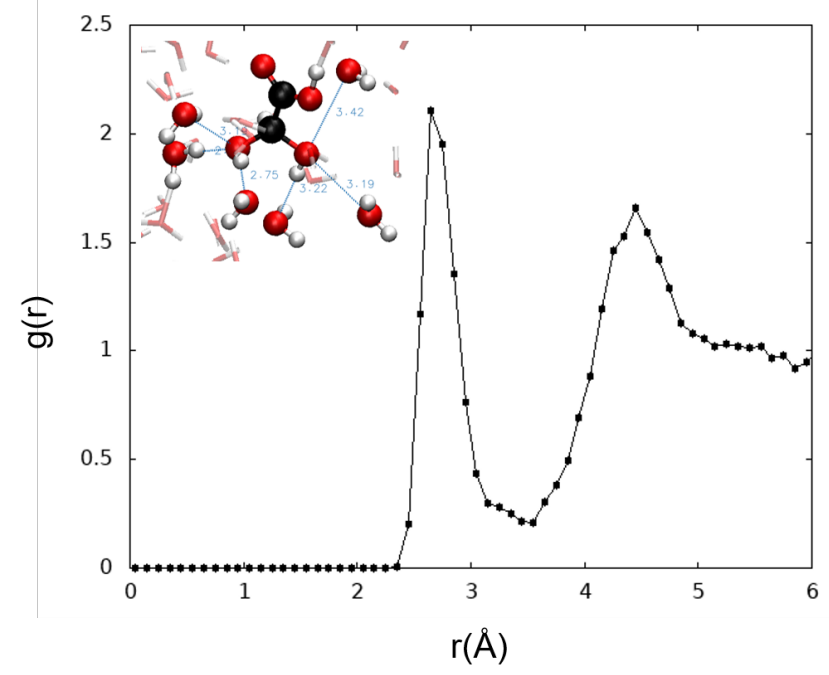

Figure 5: (Hydroxyl)oxygen-(water)oxygen RDF within the second basin (gem-diol) in the case of glyoxylic acid. X-axis units in A. Inset: A snapshot taken from the MD trajectory showing the interactions between the hydroxyls of the gem-diol and water molecules. Dashed lines indicate the distances between the oxygens of glyoxylic acid and interacting water molecules computed for the RDF calculations.

\section{Pyruvic acid: thermodynamics and structure}

The same thermodynamic and structural analysis of the calculated metadynamics trajectory was made in the case of pyruvic acid. The overall shape of the free-energy landscape (Figure 6) resembles that of glyoxylic acid, although the first basin appears wider and relatively deeper, and the localization of the transition state region is shifted (see below). The coordinates of the centers of the two basins are respectively $(0.49,0.13)$ and $(1.30,0.88)$. From this 


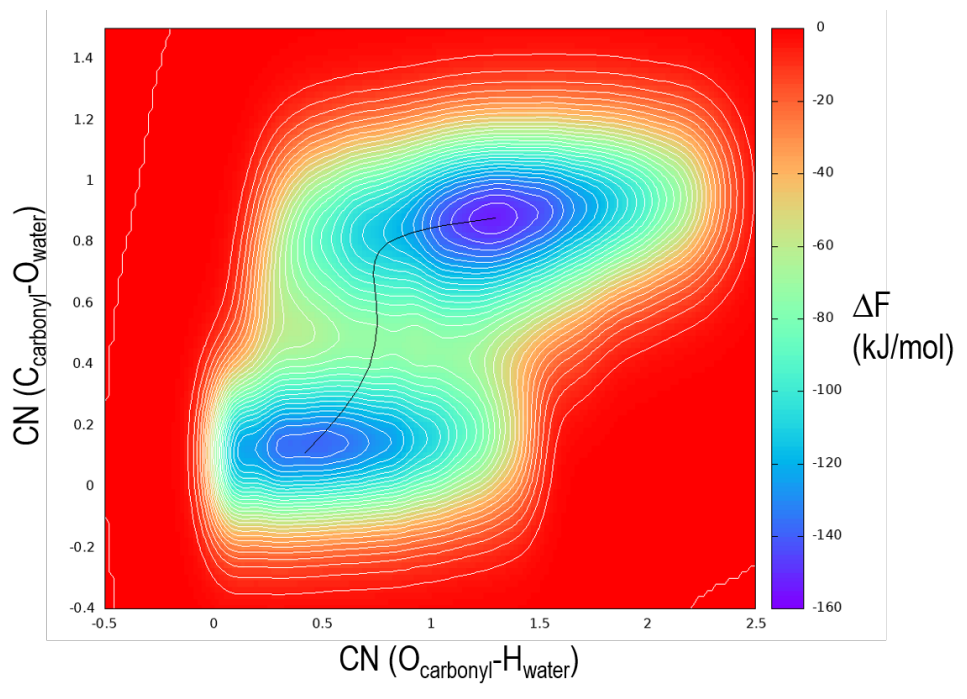

Figure 6: Free-energy (in kJ/mol) hypersurface obtained for pyruvic acid. CV1 (coordination number of $\mathrm{O}_{\text {carbonyl }}$ with respect to water hydrogens) as x-axis and CV2 (coordination number of $\mathrm{C}_{\text {carbonyl }}$ with respect to water oxgyens) as y-axis. The lowest free-energy pathway is shown.

free-energy hypersurface, the second basin is estimated to be more stable by $16 \mathrm{~kJ} / \mathrm{mol}$ (see Table 1). The energy difference between the two basins is lower than the $29 \mathrm{~kJ} / \mathrm{mol}$ found for glyoxylic acid. It is in agreement with the very different $K_{H}$ values between glyoxylic and pyruvic acids, i.e. respectively 1100 and 1.4 (preferred values in Ref. 19), indicating that the hydration is more strongly shifted toward the diol form in the former case.

The free-energy barriers to cross these minima were determined after the localisation of the transition-state around coordinates $(0.56,0.52)$, namely 68 and $84 \mathrm{~kJ} / \mathrm{mol}$. Again, in order to identify the species present, we use both visual observation of the trajectory and calculation of the (carboxyl)oxygen-(water)hydrogen RDF (Figure S2) inside these three regions, which contain 22038, 25432 and 1904 configurations, respectively. We can deduce that pyruvate anion is almost uniquely present in the first basin, whereas the gem-diol exists as a mixture of protonated and deprotonated forms in the second basin. We can also infer that in our simulation, the $\mathrm{pH}$ is larger than $\mathrm{pKa}_{\text {keto }}=1.7-2.2^{26,30-32,62}$ in the pyruvate basin while $\mathrm{pH}$ is close to $\mathrm{pKa}_{\text {diol }}=3.3-3 \cdot 6^{32,62,63}$ in the gem-diol basin.

If we compare the calculated free-energy barrier of dehydration reported in the present work $(84 \mathrm{~kJ} / \mathrm{mol}$ ) with the experimental barrier of 79 or $77 \mathrm{~kJ} / \mathrm{mol}$ (deduced using the Eyring 
equation from rate constants of $0.096,0.22$ or $0.166 s^{-1}$ at $298 \mathrm{~K}$ in acidic conditions), ${ }^{28,39,63}$ the agreement is fairly good with a slight overestimation. We did not find any value for pyruvate in literature so we refer to studies on methyl- and ethyl-pyruvate. ${ }^{28,39}$ The comparison with the barriers determined for the anionic diols, namely $81 \mathrm{~kJ} / \mathrm{mol}$ (respectively from $k_{\text {dehyd }}$ of 0.04 and $\left.0.039 \mathrm{~s}^{-1}\right)^{28}$ is even better. As for the hydration, our estimated value of $68 \mathrm{~kJ} / \mathrm{mol}$ is lower than the experimental barriers of 78 and $79 \mathrm{~kJ} / \mathrm{mol}$ measured for methyl- and ethyl-pyruvate in Ref. 28 (respectively from $k_{h y d}$ of 0.115 and $0.09 \mathrm{~s}^{-1}$ ), or than $76 \mathrm{~kJ} / \mathrm{mol}$ as found in Ref. 39 (from $k_{\text {hyd }}$ of $0.27 \mathrm{~s}^{-1}$ ).

By limiting the structural analysis within the pyruvate free-energy basin, we confirm that the anion is twisted with an average OCCO dihedral angle of -92 degrees (standard deviation of 29 degrees). We note that this value differs from that reported by Kakkar et al., namely 37 degrees when the solvent is modeled by one explicit water molecule per carboxylate oxygen and a dielectric continuum. ${ }^{33}$ In contrast, our simulation shows that each oxygen forms hydrogen bonds with two water molecules. It may explain why the twist angles differ in the two studies, again pointing the importance of an accurate solvation model as provided by the AIMD approach. Regarding the gem-diol free-energy basin, we find similar results to glyoxylic acid with both cis and trans conformations being observed for the protonated form in our simulation (Figure 7). Also, we note as previously that the same structural arrangement featuring two hydroxyl groups pointing in opposite up and down directions is observed most frequently. This can be seen on the (methyl)carbon-(hydroxyl)hydrogen RDF (Figure 8) that peaks at longer distances than for glyoxylic acid, i.e. 2.65 and $3.15 \AA$ due to the longer $\mathrm{C}-\mathrm{C}^{\alpha}$ bond in pyruvic acid. The distribution of the two peaks is also broader, which can be explained by the presence of symmetrical configurations with both hydroxyls pointing in the same direction. Also, the same hydrogen bonded arrangement as for glyoxylic acid can be observed around the hydroxyl groups, namely two donor water molecules and one acceptor water molecule, at an approximate (hydroxyl)oxygen-(water)oxygen distance of $2.65 \AA$ (Figure 9, with a number integral of 3.10 found for the first peak). This results in an 

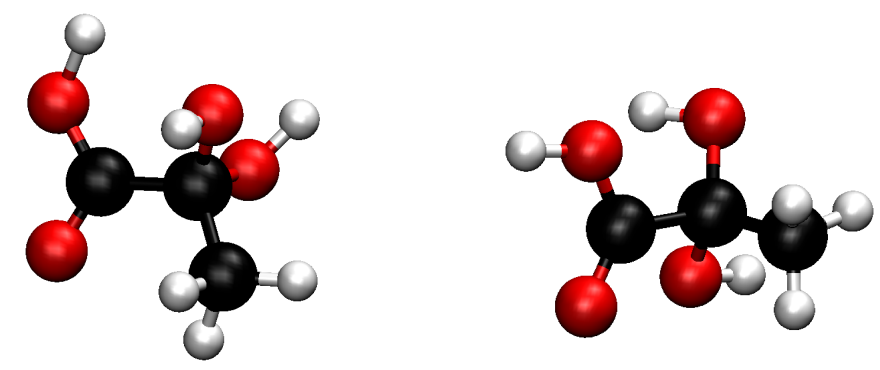

Figure 7: Configurations showing a gem-diol of pyruvic acid in cis (left) and trans (right) conformations with the two hydroxyl groups attached to $\mathrm{C}^{\alpha}$ pointing in opposite up and down directions. Solvent molecules are not shown.

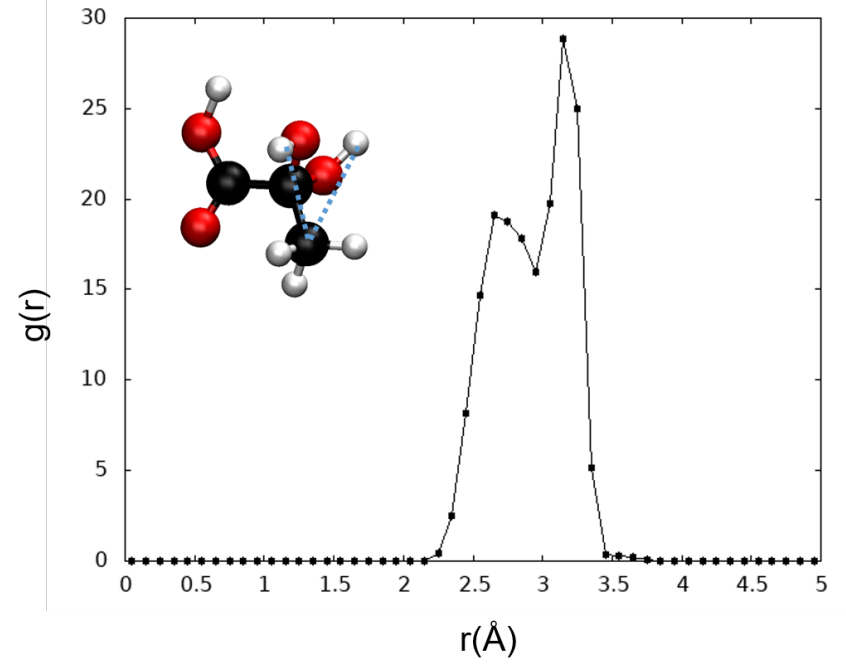

Figure 8: (Methyl)carbon-(hydroxyl)hydrogen RDF within the second basin (gem-diol) in the case of pyruvic acid. X-axis units in $\AA$. Example of a gem-diol structure as inset. Dashed lines indicate the distances computed for the RDF calculations. 
OH bond length of $1.03(0.04) \AA$. For comparison, a geometry optimization of the gem-diol in vacuo at the PBE0/def2-TZVPP level gives a bond length of $0.96 \AA$ (Figure S5 and Table S2). This again emphasizes that solvent effects have to be taken into account with, at least, a few explicit water molecules attached to these solvation sites.

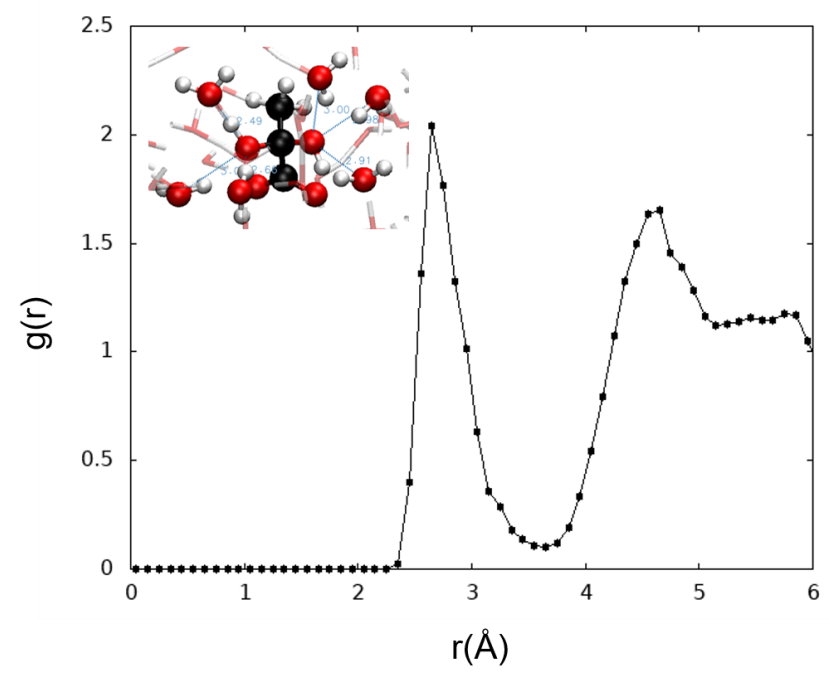

Figure 9: (Hydroxyl)oxygen-(water)oxygen RDF within second basin (gem-diol) in the case of pyruvic acid. X-axis units in A. Inset : A snapshot taken from the MD trajectory showing the interactions between the hydroxyls of the gem-diol and water molecules. Dashed lines indicate the distances between the oxygens of pyruvic acid and interacting water molecules computed for the RDF calculations.

\section{Glyoxylic and pyruvic acids: mechanisms of the reactions}

A closer look at both free-energy hypersurfaces sheds light on the mechanism of the reversible hydration of $\alpha$-oxoacids compounds. In particular, the localization and width of the transition state region is slightly different for each acid. Firstly, the width of this basin for glyoxylic acid is approximately half the width for pyruvic acid. Its center also falls at a smaller value of CV1, respectively 0.40 and 0.56 for glyoxylic and pyruvic acid (Figures 2 and 6). The minimum free-energy path that joins the first and second basins shows a steeper slope when leaving the first basin towards the transition state in the case of glyoxylic acid. This means that the first step for the hydration of the glyoxylate anion is mostly an attack 
of a water oxygen atom onto the aldehyde carbon whereas the reaction appears more concerted in the pyruvic case. One can rationalize this difference by the inductive effect of the electron donating methyl group of pyruvate (whereas there is no effect from the hydrogen of glyoxylate), which results in a less electrophilic ketone carbon, hence less prone to water oxygen attacks (CV2). This reduced reactivity can explain why the free-energy barrier for hydration is higher in the case of pyruvate $(68 \mathrm{~kJ} / \mathrm{mol})$ in comparison with glyoxylate $(52 \mathrm{~kJ} / \mathrm{mol})$. In addition, the analysis of the respective trajectories within the first basins shows that, for glyoxylate, only one hydrogen bond can be observed between the aldehyde oxygen and water, whereas for pyruvate, the ketone oxygen mostly accepts two hydrogen bonds from the solvent, which results in a more favorable water proton attack (CV1).

Concerning the water structure of the transition state, the corresponding free-energy region always includes one water molecule coordinated to the $\alpha$-carbon at an average distance of 1.87 (0.06) $\AA$ for glyoxylic acid and 1.98 (0.05) $\AA$ for pyruvic acid, which also confirms the reduced electrophilicity of the ketone carbon in the latter case. In the case of glyoxylic acid, the average angle between the water dipole and the $O_{\text {water }}-C^{\alpha}$ vector, of $62.1(9.6)$ degrees, reveals the strong interaction between one water lone electron pair and the electrophilic $\alpha$-carbon (Figure 10; this is obtained from a calculation of maximally localized Wannier functions which result from a unitary transformation of the occupied Kohn-Sham orbitals ${ }^{64}$ ). In addition, the number integral of the carbonyl-(water)oxygen RDF for second neighbors (Figures S1 and S4) is 1.55 for glyoxylic acid and 1.59 for pyruvic acid, which means that nearly two water molecules are found in the vicinity of the carbonyl group in addition to the attacking water molecule. This is in agreement with previous experimental findings of the formation of a trihydrate. ${ }^{28,39,63}$ However, our ab initio simulations in aqueous solution do not exhibit well-defined hydrogen bond networks such as rings linking the carboxyl and the carbonyl groups, in contrast to hypothetical structures pictured in experimental studies ${ }^{28,63}$ or geometry optimizations of microsolvated configurations. ${ }^{24,27,41,42,63}$ The latter obviously lack the competitive formation of hydrogen bonds with bulk water as well as their dynamics 
caused by thermal fluctuations at ambient temperature.

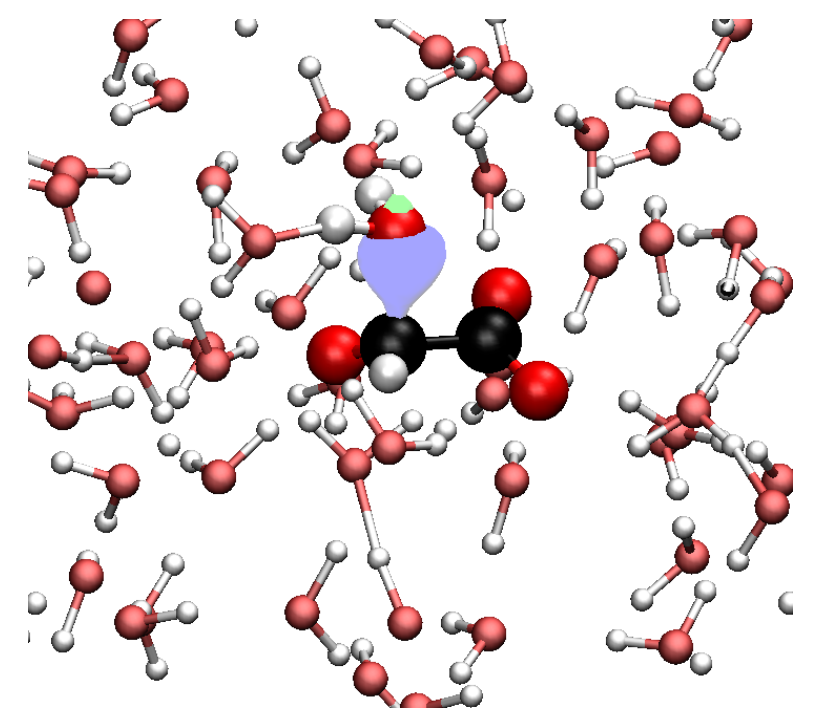

Figure 10: Typical configuration for the transition state of glyoxylic acid showing a water lone electron pair (blue and green isosurface of a maximally Wannier function) interacting with the $\alpha$-carbon.

\section{Summary and Conclusions}

The reversible hydration of glyoxylic and pyruvic acids in water was examined using ab initio molecular dynamics simulation coupled with the metadynamics method. With this powerful tool, an in silico experiment was performed where covalent bonds of the diol were reversibly formed in aqueous solution and the free-energy landscapes were reconstructed. The AIMD approach undertaken in this work agrees well with the experimental results from literature in terms of structural diversity, energetics and thermodynamics.

Three speciated forms were identified, namely neutral and anionic forms for the diol, and mainly the anionic form for the keto. Our simulations reveal that the latter is a key structure in the hydration equilibrium of glyoxylic and pyruvic acids and should therefore be more accounted for in future studies. Glyoxylate or pyruvate is highly predominant in solution due to spontaneous and rapid loss of the acidic proton, as shown by our molecular dynamics. It is in good agreement with previous experimental works reporting the higher abundance of the 
pyruvate anion. ${ }^{27,32,34}$ Both the anion and the neutral keto are active under solar radiation (above $\sim 290 \mathrm{~nm}$ ) but few studies were devoted to the photochemistry of the former despite its prominence, ${ }^{27,32,34}$ while detailed mechanisms were proposed for the latter. ${ }^{7,27,32,34}$ To the best of our knowledge, reactive pathways from the glyoxylate anion have not yet been studied and only the neutral glyoxylic acid has been investigated. ${ }^{21,65}$ From a computational point of view, the reactivity of pyruvate was examined using static, i.e. gas-phase, microsolvated pyruvate-water complexes, ${ }^{27}$ or in implicit solvent, with a limited number of explicit water molecules and a dielectric continuum. ${ }^{33}$ These approaches are relevant when focusing on specific properties, such as spectroscopic signatures. However, they may be oversimplified when trying to mimic realistic solvated structures, as we show in the present study.

The AIMD method was successful in capturing the important thermodynamic properties of the hydration equilibrium. The barriers separating the keto and gem-diol free-energy basins were calculated, a structural analysis was performed within each basin. Of importance, we were able to determine the reactive pathways connecting these basins and a new hydration mechanism was proposed. The hydration of the glyoxylate anion proceeds through a two-step process for the water addition onto the carbonyl moiety, first the attack of a water oxygen onto the carbonyl carbon then the attack of a hydrogen from a second water molecule onto the carbonyl oxygen. The reaction is more concerted in the case of pyruvate due to a less electrophilic carbonyl carbon. Our results do not confirm previous assumptions where ring structures stabilize the transition state in the aqueous phase. ${ }^{28,39}$

In aqueous solution at ambient temperature, complex and dynamic networks of hydrogen bonds affect the conformational landscape of the acids in different ways. For example, the formation of intramolecular bonds that favour the Tc conformation of glyoxylic and pyruvic acids are impeded, making the $T c$ label not relevant anymore in aqueous solution, in contrast to gas-phase conditions. A high conformational flexibility is also seen for the anionic keto. We observe that the pyruvate adopts twisted configurations, which are more highly distorted than the conformations found in microsolvated calculations ${ }^{27}$ or when using an approximated 
treatment of solvation. ${ }^{33}$ While this is beyond the scope of this study, the calculated UVvisible spectrum based on all the solvated conformers sampled during our simulations would be improved in comparison with experiment, as assumed in Ref. 27 where small discrepancies were attributed to a limited number of solvent molecules.

More precisely, this work provides a detailed description of the solvation sites of each species. The diol species can interact with up to six water molecules. Each hydroxyl moiety interacts with three water molecules, two donors plus one acceptor, and the carboxyl/carboxylate group can connect with up to three water molecules. In contrast, the keto forms are in close contact with only one or two water molecules in total. This increased hydrogen-bonding for the diol form agrees well with its higher tendency to partition more favorably to the aqueous phase than the keto form, which more likely partitions to the gas phase. ${ }^{23}$

Further improvements would provide interesting perspectives to this whole AIMD approach: (i) a better density functional approximation for a more accurate description of the subtle interplay of covalent bonds, hydrogen bonds, and van der Waals interactions, such as the SCAN functional; ${ }^{66}$ (ii) an additional collective variable within metadynamics to take into account the changing protonation state during the reactions; and (iii) simulations with an excess hydrated proton to mimic acidic aqueous solutions that will allow a more straightforward comparison with experiments. The last two items would take these atomic-scale simulations one step further since the $\mathrm{pH}$ is not directly controlled. Also, with more computational resources or more efficient algorithms, a larger simulation cell would result in less concentrated solutions.

\section{Acknowledgement}

This work was supported by "Investissements d'Avenir" LabEx PALM (ANR-10-LABX0039-PALM) and by the ANR DYNAAMIX project (ANR-17-CE29-0001-01) of the French 
Agence Nationale de la Recherche. This work was granted access to the HPC resources of CINES under the allocation DARI-A0060810750(2019) attributed by GENCI (Grand Equipement National de Calcul Intensif). The authors also thank Dr. Andrew Mayne for his careful reading of the manuscript and helpful comments.

\section{Supporting Information Available}

The (carboxyl)oxygen-(water)hydrogen RDF for first basin (black squares), second basin (white triangles), and transition state (black triangles) for glyoxylic acid (Figure S1); the (carboxyl)oxygen-(water)hydrogen RDF for first basin (black squares), second basin (white triangles), and transition state (black triangles) in the case of pyruvic acid (Figure S2); the free-energy barriers $(\mathrm{kJ} / \mathrm{mol})$ of hydration and dehydration for glyoxylic and pyruvic acids and their anion. The experimental values are obtained from the Eyring equation. Our simulations mainly describe the hydration of the anionic keto forms and the dehydration of a mixture of neutral and anionic diols (Table S1); the carbonyl-(water)oxygen RDF within transition state region for glyoxylic acid (black squares) and pyruvic acid (white triangles) (Figure S3); the number integral of carbonyl-(water)oxygen RDF within transition state region for glyoxylic acid (black squares) and pyruvic acid (white triangles) (Figure S4); the xample of a gas-phase diol form of pyruvic acid computed at the PBE0/def2-TZVPP level of theory. The $\mathrm{OH}$ bond lengths of the geminal hydroxyl groups are given (Figure S5); the cartesian coordinates of the gas-phase diol structure of pyruvic acid depicted in Figure S5 (Table S2).

\section{References}

(1) Chebbi, A.; Carlier, P. Carboxylic acids in the troposphere, occurrence, sources, and sinks: A review. Atmos. Environ. 1996, 30, 4233 - 4249. 
(2) Agarwal, S.; Aggarwal, S. G.; Okuzawa, K.; Kawamura, K. Size distributions of dicarboxylic acids, ketoacids, $\alpha$-dicarbonyls, sugars, WSOC, OC, EC and inorganic ions in atmospheric particles over Northern Japan: Implication for long-range transport of Siberian biomass burning and East Asian polluted aerosols. Atmos. Chem. Phys. 2010, $10,5839-5858$.

(3) Kanakidou, M.; Seinfeld, J. H.; Pandis, S. N.; Barnes, I.; Dentener, F. J.; Facchini, M. C.; Van Dingenen, R.; Ervens, B.; Nenes, A.; Nielsen, C. J. et al. Organic aerosol and global climate modelling: a review. Atmos. Chem. Phys. 2005, 5, 10531123.

(4) Huff Hartz, K. E.; Tischuk, J. E.; Chan, M. N.; Chan, C. K.; Donahue, N. M.; Pandis, S. N. Cloud condensation nuclei activation of limited solubility organic aerosol. Atmos. Environ. 2006, 40, 605-617.

(5) Zhang, R.; Wang, G.; Guo, S.; Zamora, M.; Ying, Q.; Lin, Y.; Wang, W.; Hu, M.; Wang, Y. Formation of Urban Fine Particulate Matter. Chem. Rev. 2012, 115, $3803-3855$.

(6) Löflund, M.; Kasper-Giebl, A.; Schuster, B.; Giebl, H.; Hitzenberger, R.; Puxbaum, H. Formic, acetic, oxalic, malonic and succinic acid concentrations and their contribution to organic carbon in cloud water. Atmos. Environ. 2002, 36, 1553-1558.

(7) Guzmàn, M. I.; Colussi, A. J.; Hoffmann, M. R. Photoinduced Oligomerization of Aqueous Pyruvic Acid. J. Phys. Chem. A 2006, 110, 3619-3626.

(8) Kawamura, K.; Tachibana, E.; Okuzawa, K.; Aggarwal, S. G.; Kanaya, Y.; Wang, Z. F. High abundances of water-soluble dicarboxylic acids, ketocarboxylic acids and $\alpha$ dicarbonyls in the mountaintop aerosols over the North China Plain during wheat burning season. Atmos. Chem. Phys. 2013, 13, 8285-8302. 
(9) Fu, P.; Kawamura, K.; Usukura, K.; Miura, K. Dicarboxylic acids, ketocarboxylic acids and glyoxal in the marine aerosols collected during a round-the-world cruise. Mar. Chem. 2013, 148, 22-32.

(10) Carlton, A. G.; Turpin, B. J.; Lim, H.-J.; Altieri, K. E.; Seitzinger, S. Link between isoprene and secondary organic aerosol (SOA): Pyruvic acid oxidation yields low volatility organic acids in clouds. Geophys. Res. Lett. 2006, 33, L06822.

(11) Altieri, K. E.; Carlton, A. G.; Lim, H.-J.; Turpin, B. J.; Seitzinger, S. P. Evidence for Oligomer Formation in Clouds: Reactions of Isoprene Oxidation Products. Environ. Sci. Technol. 2006, 40, 4956-4960.

(12) George, C.; Ammann, M.; D’Anna, B.; Donaldson, D. J.; Nizkorodov, S. A. Heterogeneous Photochemistry in the Atmosphere. Chem. Rev. 2015, 115, 4218-4258.

(13) Herrmann, H.; Schaefer, T.; Tilgner, A.; Styler, S. A.; Weller, C.; Teich, M.; Otto, T. Tropospheric Aqueous-Phase Chemistry: Kinetics, Mechanisms, and Its Coupling to a Changing Gas Phase. Chem. Rev. 2015, 115, 4259-4334.

(14) Pöschl, U.; Shiraiwa, M. Multiphase Chemistry at the Atmosphere-Biosphere Interface Influencing Climate and Public Health in the Anthropocene. Chem. Rev. 2015, 115, $4440-4475$.

(15) Reed Harris, A. E.; Pajunoja, A.; Cazaunau, M.; Gratien, A.; Pangui, E.; Monod, A.; Griffith, E. C.; Virtanen, A.; Doussin, J.-F.; Vaida, V. Multiphase Photochemistry of Pyruvic Acid under Atmospheric Conditions. J. Phys. Chem. A 2017, 121, 3327-3339.

(16) Tan, Y.; Lim, Y. B.; Altieri, K. E.; Seitzinger, S. P.; Turpin, B. J. Mechanisms leading to oligomers and $\mathrm{SOA}$ through aqueous photooxidation: insights from $\mathrm{OH}$ radical oxidation of acetic acid and methylglyoxal. Atmos. Chem. Phys. 2012, 12, 801-813. 
(17) Liu, Y.; Monod, A.; Tritscher, T.; Praplan, A. P.; DeCarlo, P. F.; Temime-Roussel, B.; Quivet, E.; Marchand, N.; Dommen, J.; Baltensperger, U. Aqueous phase processing of secondary organic aerosol from isoprene photooxidation. Atmos. Chem. Phys. 2012, 12, 5879-5895.

(18) Charbouillot, T.; Gorini, S.; Voyard, G.; Parazols, M.; Brigante, M.; Deguillaume, L.; Delort, A.-M.; Mailhot, G. Mechanism of carboxylic acid photooxidation in atmospheric aqueous phase: Formation, fate and reactivity. Atmos. Environ. 2012, 56, 1 - 8.

(19) Doussin, J.-F.; Monod, A. Structure-activity relationship for the estimation of OHoxidation rate constants of carbonyl compounds in the aqueous phase. Atmos. Chem. Phys. 2013, 13, 11625-11641.

(20) Mkoma, S. L.; Kawamura, K. Molecular composition of dicarboxylic acids, ketocarboxylic acids, $\alpha$-dicarbonyls and fatty acids in atmospheric aerosols from Tanzania, East Africa during wet and dry seasons. Atmos. Chem. Phys. 2013, 13, 2235-2251.

(21) Xia, S.-S.; Eugene, A. J.; Guzman, M. I. Cross Photoreaction of Glyoxylic and Pyruvic Acids in Model Aqueous Aerosol. J. Phys. Chem. A 2018, 122, 6457-6466.

(22) Chen, C.; Shyu, S.-F. Theoretical study of glyoxylic and pyruvic acids: rotamers and intramolecular hydrogen bonding. J. Mol. Struct. Theochem 2000, 503, 201-211.

(23) Plath, K. L.; Axson, J. L.; Nelson, G. C.; Takahashi, K.; T., S. R.; Vaida, V. Gas-phase vibrational spectra of glyoxylic acid and its gem diol monohydrate. Implications for atmospheric chemistry. React. Kinet. Catal. Lett. 2009, 96, 209-224.

(24) Lundell, J.; Olbert-Majkut, A. Isolated glyoxylic acid-water 1:1 complexes in low temperature argon matrices. Spectrochim. Acta A Mol. Biomol. Spectrosc. 2015, 136, 113121. 
(25) Reva, I.; Nunes, C. M.; Biczysko, M.; Fausto, R. Conformational Switching in Pyruvic Acid Isolated in Ar and N2 Matrixes: Spectroscopic Analysis, Anharmonic Simulation, and Tunneling. J. Phys. Chem. A 2015, 119, 2614-2627.

(26) Lopalco, A.; Douglas, J.; Denora, N.; Stella, V. J. Determination of pKa and Hydration Constants for a Series of $\alpha$-Keto-Carboxylic Acids Using Nuclear Magnetic Resonance Spectrometry. J. Pharm. Sci. 105, 664-672.

(27) Shemesh, D.; Luo, M.; Grassian, V. H.; Gerber, R. B. Absorption spectra of pyruvic acid in water: insights from calculations for small hydrates and comparison to experiment. Phys. Chem. Chem. Phys. 2020, 22, 12658-12670.

(28) Sørensen, P. E.; Bruhn, K.; Lindeløv, F. Kinetics and Equilibria for the Reversible Hydration of the Aldehyde Group in Glyoxylic Acid. Acta Chem. Scand. Ser. A Phys. Inorg. Chem. 1974, 28a, 162-168.

(29) Olson, T. M.; Hoffmann, M. R. Formation kinetics, mechanism, and thermodynamics of glyoxylic acid-sulfur(IV) adducts. J. Phys. Chem. 1988, 92, 4246-4253.

(30) Hellström, N.; Almqvist, S.-O. Hydration and dehydration of pyruvic acid. J. Chem. Soc. B 1970, 1396-1400.

(31) Pocker, Y.; Meany, J. E.; Nist, B. J.; Zadorojny, C. Reversible hydration of pyruvic acid. I. Equilibrium studies. J. Phys. Chem. 1969, 73, 2879-2882.

(32) Rapf, R. J.; Dooley, M. R.; Kappes, K.; Perkins, R. J.; Vaida, V. pH Dependence of the Aqueous Photochemistry of $\alpha$-Keto Acids. The Journal of Physical Chemistry A 2017, 121, 8368-8379, PMID: 29032688.

(33) Kakkar, R.; Pathak, M.; Gahlot, P. Effect of aqueous solvation on the structures of pyruvic acid isomers and their reactions in solution: a computational study. J. Phys. Org. Chem. 2008, 21, 23-29. 
(34) Rapf, R. J.; Perkins, R. J.; Carpenter, B. K.; Vaida, V. Mechanistic Description of Photochemical Oligomer Formation from Aqueous Pyruvic Acid. J. Phys. Chem. A 2017, 121, 4272-4282.

(35) Strehlow, H. Die Kinetik der Hydratation von $\alpha$-Ketocarbonsäuren. Z. Elektrochem. 1962, 66, 392-396.

(36) Becker, M. Über magnetische Kernresonanzspektren wäßriger Brenztraubensäurelösungen. Berich. Bunsen Gesell. 1964, 68, 669-676.

(37) Pocker, Y.; Meany, J. E.; Zadorojny, C. Reversible hydration of pyruvate esters. Thermodynamic and kinetic studies. J. Phys. Chem. 1971, 75, 792-799.

(38) Buschmann, H.-J.; Füldner, H.-H.; Knoche, W. The Reversible Hydration of Carbonyl Compounds in Aqueous Solution. Part I, The Keto/Gem-diol Equilibrium. Ber. Bunsen Phys. Chem. 1980, 84, 41-44.

(39) Buschmann, H.-J.; Dutkiewicz, E.; Knoche, W. The Reversible Hydration of Carbonyl Compounds in Aqueous Solution Part II: The Kinetics of the Keto/Gem-diol Transition. Ber. Bunsen. Phys. Chem. 1982, 86, 129-134.

(40) Yang, D.; Zhang, L. Excited-state hydrogen bonding dynamics of pyruvic acid and geminal-diol, 2,2-dihydroxypropanoic acid in aqueous solution: a DFT/TDDFT study. J. Phys. Org. Chem. 2013, 25, 1391-1394.

(41) Maroń, M. K.; Takahashi, K.; Shoemaker, R. K.; Vaida, V. Hydration of pyruvic acid to its geminal-diol, 2,2-dihydroxypropanoic acid, in a water-restricted environment. Chem. Phys. Lett. 2011, 513, $184-190$.

(42) Schnitzler, E. G.; Seifert, N. A.; Ghosh, S.; Thomas, J.; Xu, Y.; Jäger, W. Hydration of the simplest $\alpha$-keto acid: a rotational spectroscopic and ab initio study of the pyruvic acid-water complex. Phys. Chem. Chem. Phys. 2017, 19, 4440-4446. 
(43) Blair, S. L.; Reed Harris, A. E.; Frandsen, B. N.; Kjaergaard, H. G.; Pangui, E.; Cazaunau, M.; Doussin, J.-F.; Vaida, V. Conformer-Specific Photolysis of Pyruvic Acid and the Effect of Water. The Journal of Physical Chemistry A 2020, 124, 1240-1252.

(44) Marx, D.; Hutter, J. Ab initio Molecular Dynamics: Basic Theory and Advanced Methods; Cambridge University Press: Cambridge, 2009.

(45) Mugnai, M.; Cardini, G.; Schettino, V.; Nielsen, C. Ab initio molecular dynamics study of aqueous formaldehyde and methanediol. Mol. Phys. 2007, 105, 2203-2210.

(46) Meijer, E. J.; Sprik, M. Ab Initio Molecular Dynamics Study of the Reaction of Water with Formaldehyde in Sulfuric Acid Solution. J. Am. Chem. Soc. 1998, 120, 6345-6355.

(47) Car, R.; Parrinello, M. Unified Approach for Molecular Dynamics and DensityFunctional Theory. Phys. Rev. Lett. 1985, 55, 2471-2474.

(48) Perdew, J. P.; Burke, K.; Ernzerhof, M. Generalized Gradient Approximation Made Simple. Phys. Rev. Lett. 1996, 77, 3865-3868, Erratum: (1997) Phys Rev Lett 78:1396.

(49) Vanderbilt, D. Soft self-consistent pseudopotentials in a generalized eigenvalue formalism. Phys. Rev. B 1990, 41, 7892-7895.

(50) Roy, S.; Baer, M. D.; Mundy, C. J.; Schenter, G. K. Reaction Rate Theory in Coordination Number Space: An Application to Ion Solvation. The Journal of Physical Chemistry C 2016, 120, 7597-7605.

(51) Bussi, G.; Laio, A.; Tiwary, P. In Handbook of Materials Modeling: Methods: Theory and Modeling; Andreoni, W., Yip, S., Eds.; Springer International Publishing: Cham, 2020; pp 565-595.

(52) Bussi, G.; Laio, A. Using metadynamics to explore complex free-energy landscapes. Nat. Rev. Phys. 2020, 2, 200-212. 
(53) Bussi, G.; Branduardi, D. Reviews in Computational Chemistry Volume 28; John Wiley \& Sons, Ltd, 2015; Chapter 1, pp 1-49.

(54) Laio, A.; Gervasio, F. L. Metadynamics: a method to simulate rare events and reconstruct the free energy in biophysics, chemistry and material science. Rep. Prog. Phys. 2008, 71, 126601.

(55) Ensing, B.; Laio, A.; Parrinello, M.; Klein, M. L. A Recipe for the Computation of the Free Energy Barrier and the Lowest Free Energy Path of Concerted Reactions. J. Phys. Chem. B 2005, 109, 6676-6687.

(56) Laio, A.; Parrinello, M. Proc. Natl. Acad. Sci. USA 2002, 99, 12562-12566.

(57) Stirling, A.; Iannuzzi, M.; Laio, A.; Parrinello, M. Azulene-to-Naphthalene Rearrangement: The Car-Parrinello Metadynamics Method Explores Various Reaction Mechanisms. ChemPhysChem 2004, 5, 1558-1568.

(58) Iannuzzi, M.; Laio, A.; Parrinello, M. Efficient Exploration of Reactive Potential Energy Surfaces Using Car-Parrinello Molecular Dynamics. Phys. Rev. Lett. 2003, 90, 238302.

(59) Muñoz-Santiburcio, D.; Marx, D. Chemistry in nanoconfined water. Chem. Sci. 2017, $8,3444-3452$.

(60) Laio, A.; Rodriguez-Fortea, A.; Gervasio, F. L.; Ceccarelli, M.; Parrinello, M. Assessing the Accuracy of Metadynamics. J. Phys. Chem. B 2005, 109, 6714-6721.

(61) Gillan, M. J.; Alfè, D.; Michaelides, A. Perspective: How good is DFT for water? J. Chem. Phys. 2016, 144, 130901.

(62) Luo, M.; Shemesh, D.; Sullivan, M. N.; Alves, M. R.; Song, M.; Gerber, R. B.; Grassian, V. H. Impact of $\mathrm{pH}$ and $\mathrm{NaCl}$ and $\mathrm{CaCl} 2$ Salts on the Speciation and Photochemistry of Pyruvic Acid in the Aqueous Phase. The Journal of Physical Chemistry A 2020, 124, 5071-5080. 
(63) Strehlow, H. Relaxation techniques in fast reaction kinetics. Adv. Mol. Relaxation Processes 1972, 2, 235-249.

(64) Marzari, N.; Vanderbilt, D. Maximally localized generalized Wannier functions for composite energy bands. Phys. Rev. B 1997, 56, 12847-12865.

(65) Eugene, A. J.; Xia, S.-S.; Guzman, M. I. Aqueous Photochemistry of Glyoxylic Acid. The Journal of Physical Chemistry A 2016, 120, 3817-3826.

(66) Chen, M.; Ko, H.-Y.; Remsing, R. C.; Calegari Andrade, M. F.; Santra, B.; Sun, Z.; Selloni, A.; Car, R.; Klein, M. L.; Perdew, J. P. et al. Ab initio theory and modeling of water. Proc. Natl. Acad. Sci. USA 2017, 114, 10846-10851. 
Graphical TOC Entry

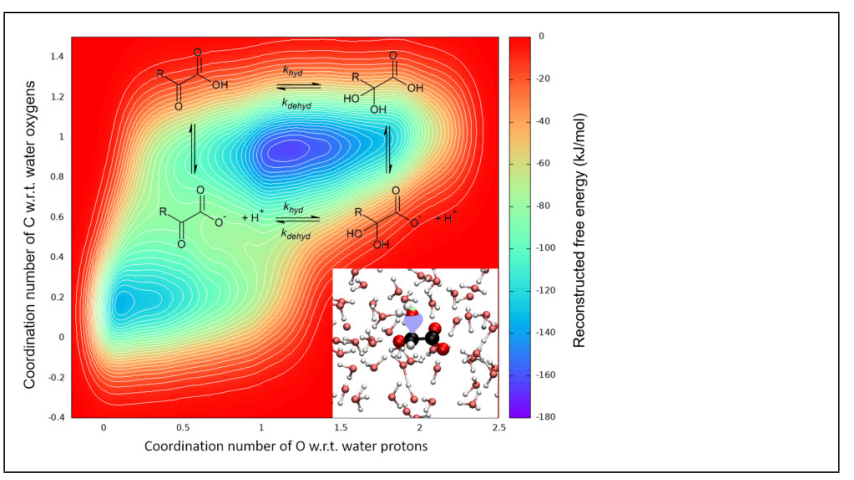

